\title{
Relationship between Happiness, Perceived Social Support and Self-Esteem with Sexual Addiction among Couples
}

\author{
Hamid Reza Samadifard ${ }^{1, *}$, iD, Niloufar Mikaeili ${ }^{2}$, Seyfollah Aghajani ${ }^{3}$
}

${ }^{1} \mathrm{PhD}$ Student of Psychology, Faculty of Educational Sciences and Psychology, University of Mohaghegh Ardabili, Ardabil, Iran

${ }^{2}$ Associate Professor, Department of Psychology, Faculty of Educational Sciences and Psychology, University of Mohaghegh Ardabili, Ardabil, Iran

${ }^{3}$ Assistant Professor, Department of Psychology, Faculty of Educational Sciences and Psychology, University of Mohaghegh Ardabili, Ardabil, Iran

* Corresponding author: Hamid Reza Samadifard, PhD Student of Psychology, Faculty of Educational Sciences and Psychology, University of Mohaghegh Ardabili, Ardabil, Iran. E-mail: hrsamadifard@uma.ac.ir

Received: 10 Nov 2017

Accepted: 10 Nov 2018

Abstract
Introduction: Sexual addiction is a growing concern known as a serious disorder. The present study was
conducted to determine the relationship between happiness, perceived social support and self-esteem with
sexual addiction among couples in Ardabil city.
Methods: The study was based on a descriptive correlational method. Its statistical population consisted of
all couples in Ardabil city in 2016 . A total of 110 people ( 55 married spouses) were selected as the statistical
sample using the convenience sampling. To collect data, the Oxford happiness scale, perceived social support
scale, self-esteem scale and sexual addiction scale were used. Research data were analyzed using Independent
t test, Pearson correlation coefficient and multiple regression.
Results: The study results indicated no significant difference in sexual addiction of men and women
(P > 0.05). Additionally, there was a significant relationship between happiness, perceived social support,
self-esteem and sexual addiction in couples (p<0.05). The results showed that happiness, perceived social
support and self-esteem could predict the sexual addiction of couples by 0.61 (P < 0.05 ).
Conclusions: It can be concluded that happiness, perceived social support and self-esteem could decrease
the sexual addiction in couples.
Keywords: Happiness, Social Support, Self-Concept, Sexual Addiction, Family Characteristics
O) 2018 Iranian Nursing Scientific Association (INA)




\section{رابطه شادكامى، حمايت اجتماعى ادراك شده و مزت نقس با اعتياد جنسى در بين زوجين}

\section{حميدرضا صمدىفرد ا.*.(i)، نيلوفر ميكائيلى '، سيفاله آقاجانى ‘}

' دانشجوى دكتراى تخصصى روانشناسى، دانشكده علوم تربيتى و روانشناسى، دانشكاه محقّق اردبيلى، اردبيل، ايران

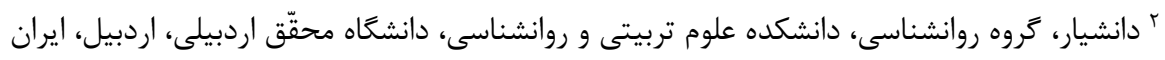

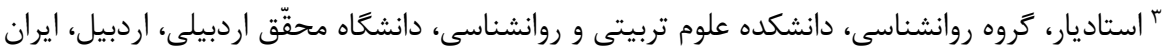

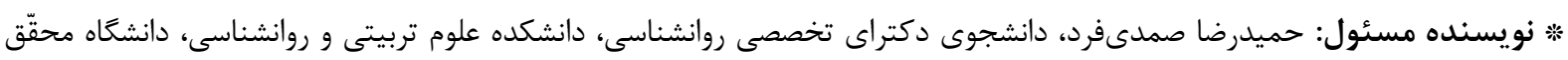
اردبيلى، اردبيل، ايران. ايميل: hrsamadifard@uma.ac.ir

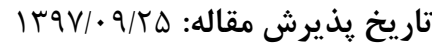

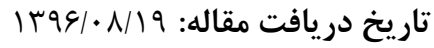

مقدمه: اعتياد جنسى يك نكرانى رو به رشد است كه به عنوان يك بيمارى جدى شناخته مىشود. يزوهش حاضر با هدف بررسى رابطه

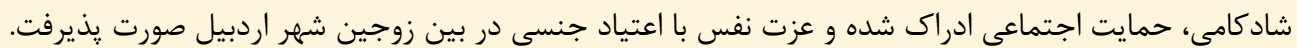

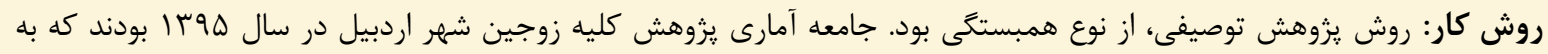

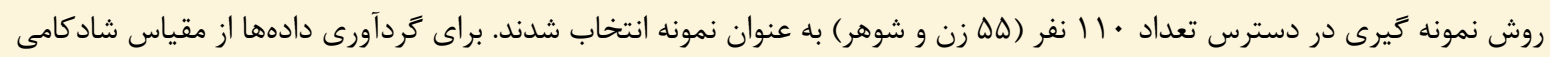

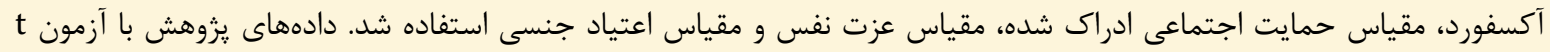

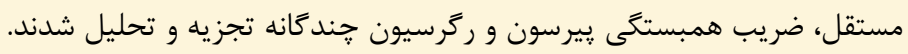

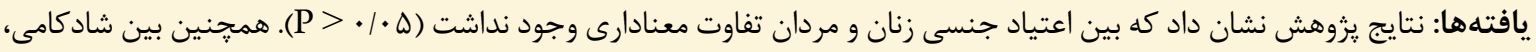

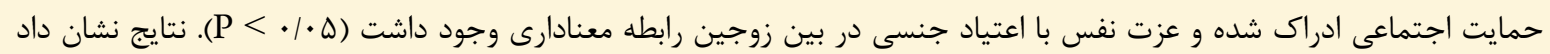

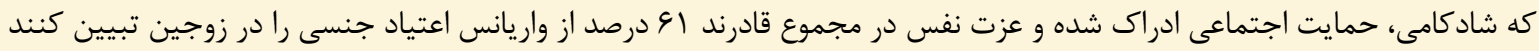
$(\mathrm{P}<\cdot / \cdot \Delta)$ نتيجه گيرى: مى توان نتيجه كرفت كه شادكامى، حمايت اجتماعى ادراك شده و عزت نفس باعث كاهش اعتياد جنسى در بين زوجين مىشود. وازگًان كليدى: شادكامى، حمايت اجتماعى ادراك شده، عزت نفس، اعتياد جنسى، زوجين

است و روابط خانوادكى را نابود مى كند [ه]]. شيوع اختلال جنسى در

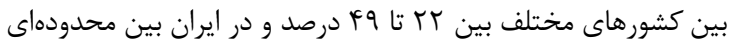

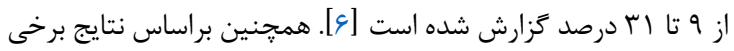

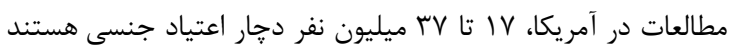

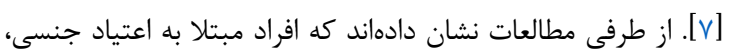

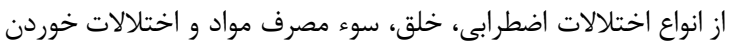

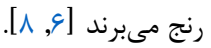

از عواملى كه مىتوانند در اعتياد جنسى زوجين تأثير خذار باشند،

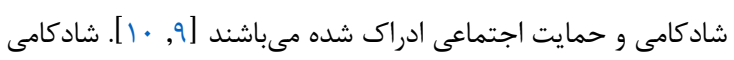

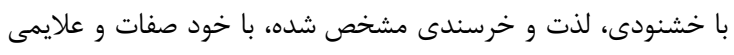

مقدمه هدف از زندكى مشترك، برطرف شدن نيازهاى زوجين است و اخر

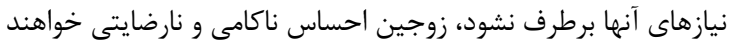

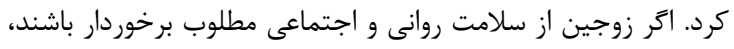

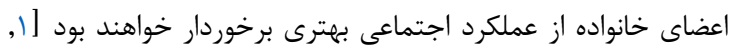

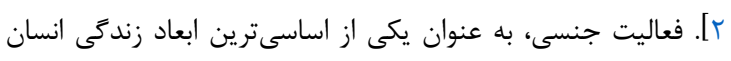

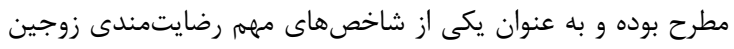

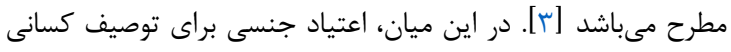

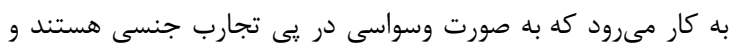

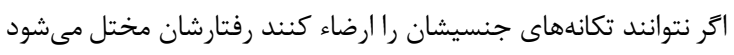

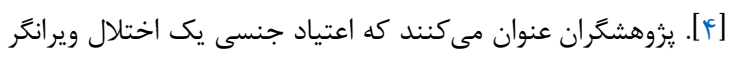


هوץT بودند كه براساس تعداد متغيرهاى يزوهش از طريق نرمافزار

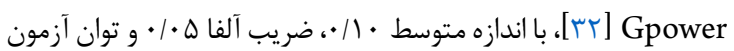

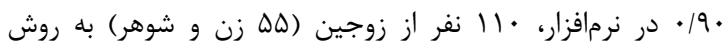

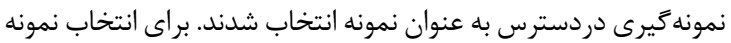

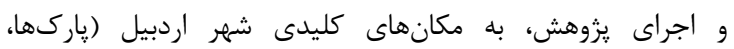

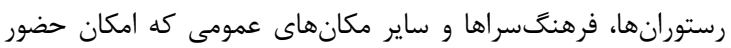

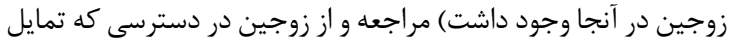

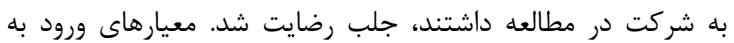

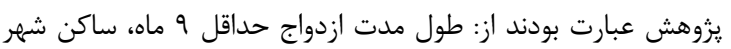

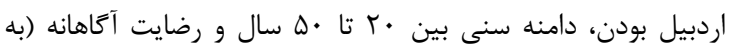

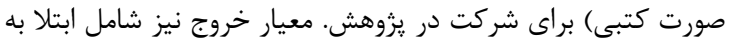

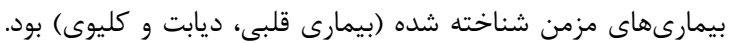

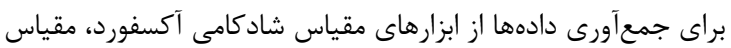

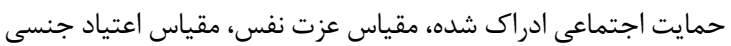

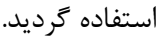

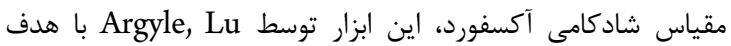

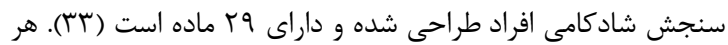

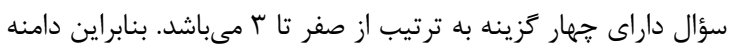

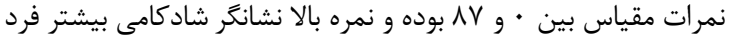

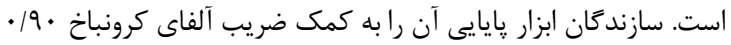

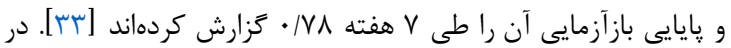

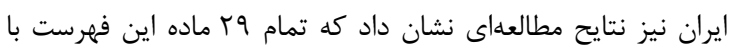

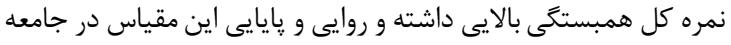

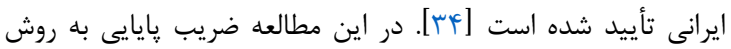

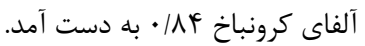

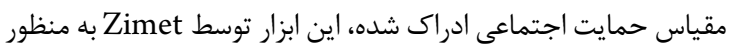

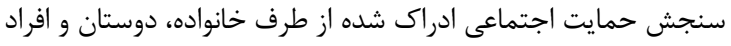

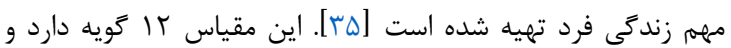

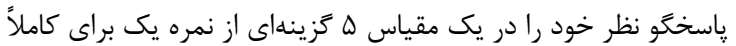

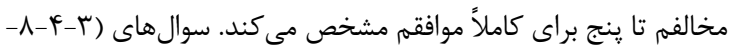

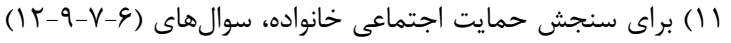

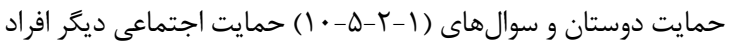

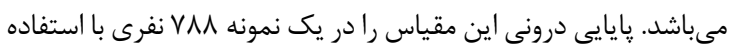

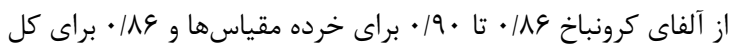

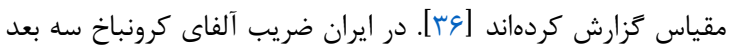

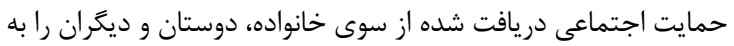

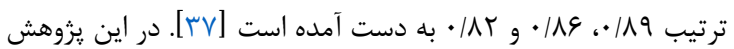

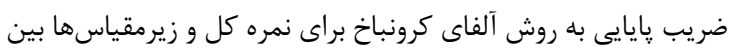

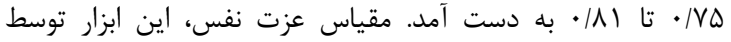

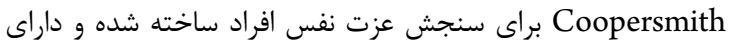

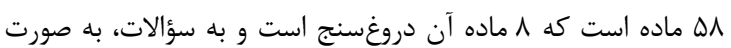

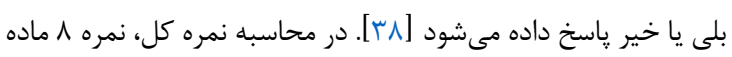

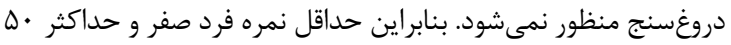

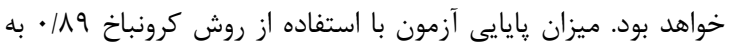

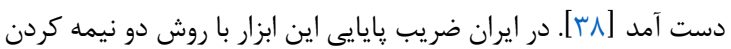

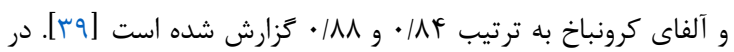

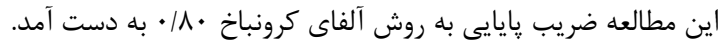

از جمله هيجانات و باورهاى مثبت به همراه داشته و داراى دو مؤلفه

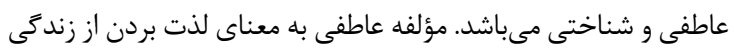

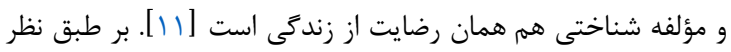

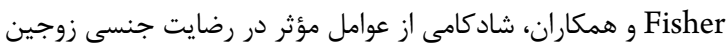

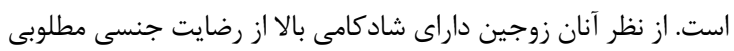

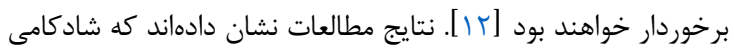

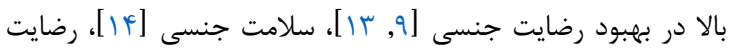

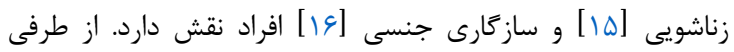

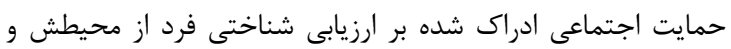

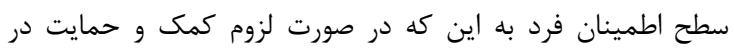

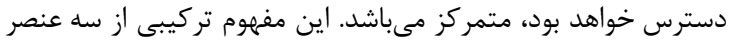

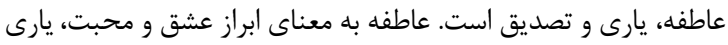

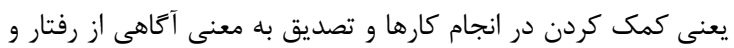

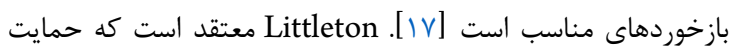
اجتماعى ادراك شده از عوامل تأثير كذار در عملكرد جنسى الفال الفراد است

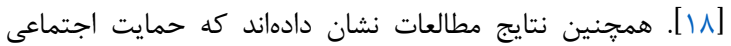

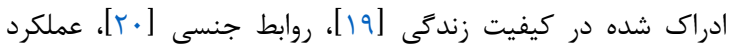

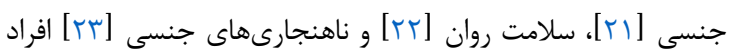

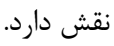
از عوامل ديكرى كه مى تواند در اعتياد جنسى زوجين تأثير كذار باشد،

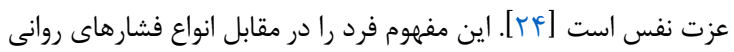

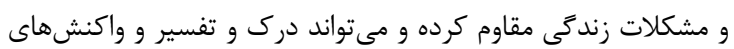

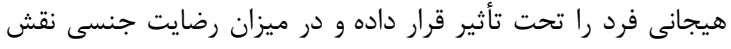

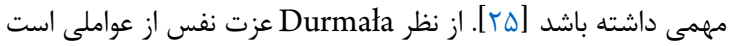

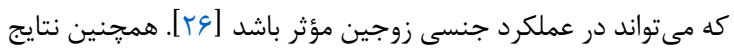

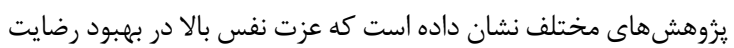

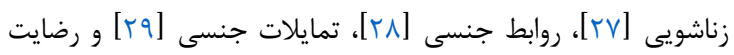

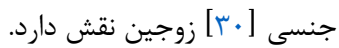

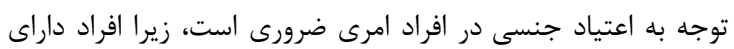

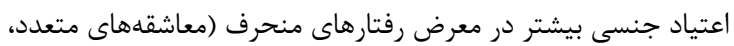

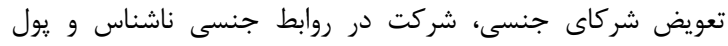

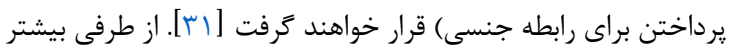

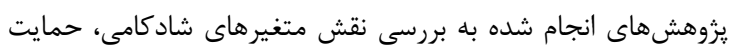

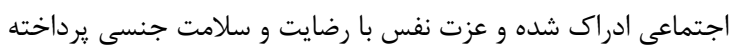

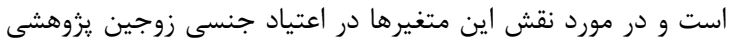

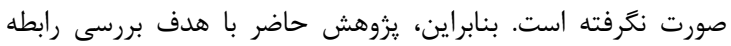

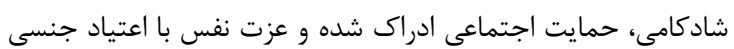

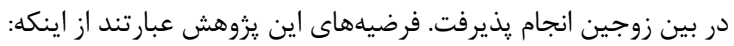

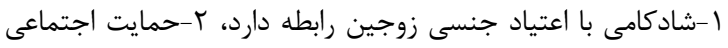

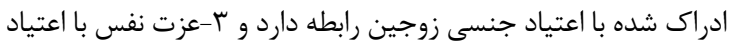
جنسى زوجين رابطه دارد.

\section{روش كار}

روش اجراى اين مطالعه توصيفى و از نوع همبستَّى بود. در اين

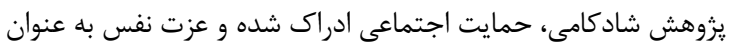

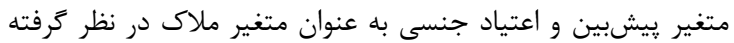

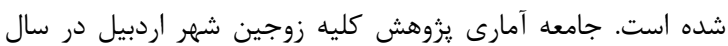


مطلوب مقياس مى باشد [ه]]. در يروهش حاضر ضريب بإيايى به روش

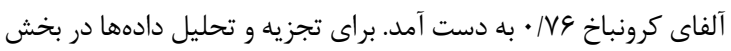

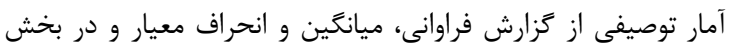

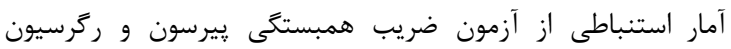

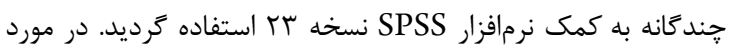

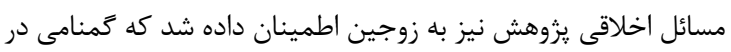

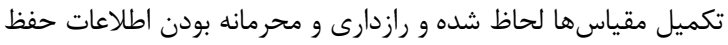

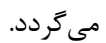

\section{يافتهها}

تعداد • ل11 انفر از زوجين شهر اردبيل در اين مطالعه شركت داده شدند.

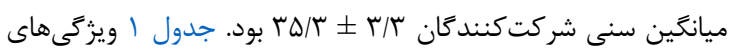
جمعيتشناختى شركت كنندكان در يروهش ران انشان مى دهد.

مقياس اعتياد جنسى، اين ابزار توسط Carnes براى سنجش اعتياد به

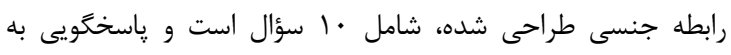

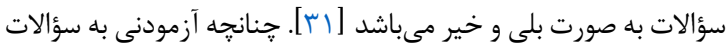

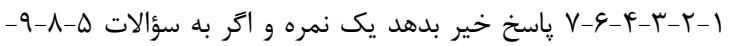

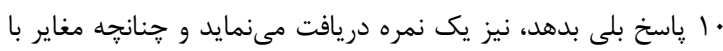

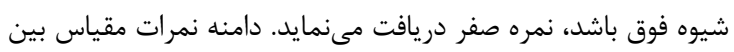

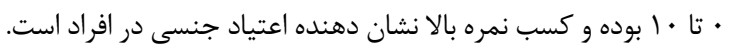

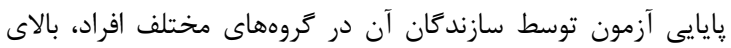

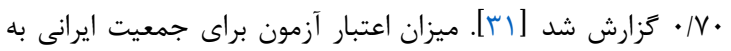

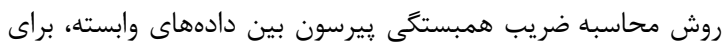

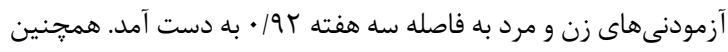

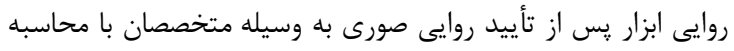
همبستخى ييرسون VT/ • كزارش شده است كه حاكى از روايى و اعتبار

جدول ا: مشخصات دموكر افيك افراد شركت كننده در مطالعه

\begin{tabular}{|c|c|c|}
\hline $\mathbf{P}$ & $\mathbf{F}$ & شاخص \\
\hline & & تحصيلات \\
\hline $\mid Q / 4$ & IV & سيكل \\
\hline$r V / r$ & il & دييلم \\
\hline \multirow[t]{2}{*}{$F V / r$} & $\Delta T$ & دانشكاهى \\
\hline & & وضعيت اقتصادى \\
\hline 19 & rI & خوب \\
\hline$\Delta q$ & 90 & متوسط \\
\hline \multirow[t]{2}{*}{ rr } & re & ضعيف \\
\hline & & وضعيت فرزند \\
\hline gr & 91 & داراى فرزند \\
\hline \multirow[t]{2}{*}{$r \Lambda$} & er & بدون فرزند \\
\hline & & طول مدت ازدواج \\
\hline$r \omega / \Delta$ & rq & $9-1$ \\
\hline$F F / D$ & $4 q$ & $19-1$. \\
\hline$r$. & rT & • r سال و بالاتر \\
\hline
\end{tabular}

در كام بعدى تحليل دادهها، يس از تأييد همكنى واريانسها با استفاده

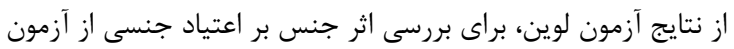

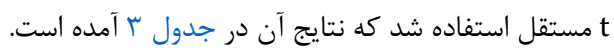

\begin{tabular}{|c|c|c|c|}
\hline Sig & $\mathbf{t}$ & درجه آزادى & متغير \\
\hline - /4TE &.$- / \mathrm{Q} 9$ & $1 \cdot 1$ & اعتياد جنسى \\
\hline
\end{tabular}

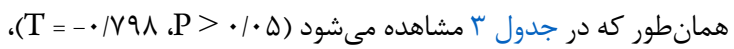

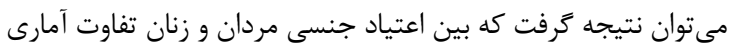

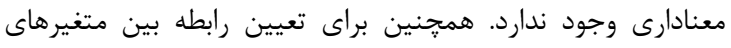

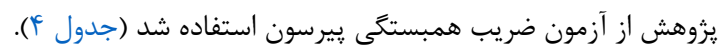

جدول r آمارههاى توصيفى متغيرهاى يزوهش (شادكامى، حمايت

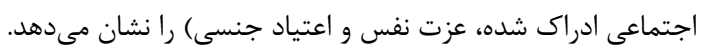

\begin{tabular}{|c|c|}
\hline ميانكين (انحراف استاندارد) & 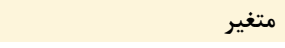 \\
\hline \multirow[t]{2}{*}{$\kappa+/ \Lambda \pm \Lambda / \Lambda$} & شادكامى \\
\hline & حمايت اجتماعى ادراك شده \\
\hline $\mid r / r \pm r / \Lambda$ & خانواده \\
\hline $11 / r \pm r$ & 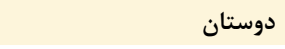 \\
\hline $11 / r \pm N / r$ & ديكر افراد \\
\hline$T V / \Delta \pm V / \Lambda$ & عزت نفس \\
\hline$\Delta / r \pm \Delta / 1$ & اعتياد جنسى \\
\hline
\end{tabular}

\begin{tabular}{|c|c|c|c|c|c|c|c|}
\hline \multirow[b]{2}{*}{9} & \multirow[b]{2}{*}{$\Delta$} & \multirow[b]{2}{*}{ F } & \multirow[b]{2}{*}{$r$} & \multirow[b]{2}{*}{$r$} & & \multicolumn{2}{|c|}{ جدول f: ماتريس همبستًى متغيرهاى يُروهش } \\
\hline & & & & & 1 & & متغ \\
\hline & & & & & - & شادكامى & 1 \\
\hline & & & & - &.$/ 49$ & 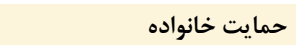 & r \\
\hline & & & - & $\cdot|9|$ & $\cdot / 4 \Delta$ & حمايت دوستان & $r$ \\
\hline & & - & $\cdot / \omega$ & $\cdot / \Delta \Delta$ & $\cdot / 4 q$ & حمايت ديكر افراد & f \\
\hline & - & $\cdot / \mu F$ & $\cdot \pi \cdot$ & . & $\cdot / r q$ & 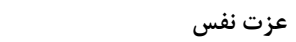 & $\Delta$ \\
\hline - & $-\cdot / \pi)$ &.$- / 49$ &.$- / 01$ &.$- / 49$ &.$- / \Delta F$ & اعتياد جنسى & q \\
\hline
\end{tabular}


نفس) از آزمون ركرسيون جندكانه به روش همزمان استفاده شد

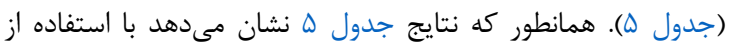

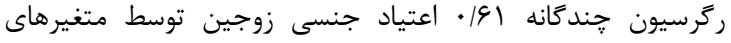

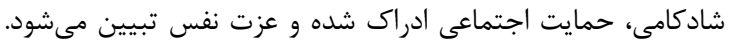

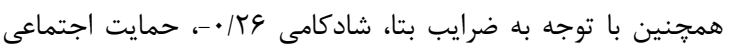

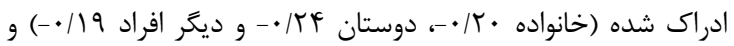

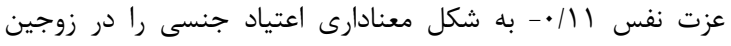

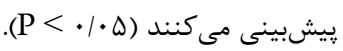

طبق نتايج جدول أ، بين شادكامى و عزت نفس با مؤلفههاى حمايت

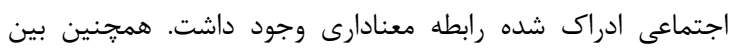

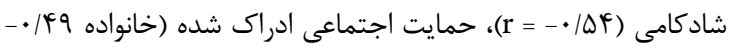

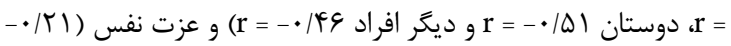

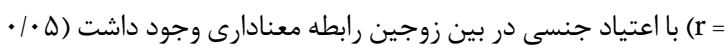

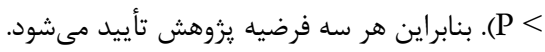

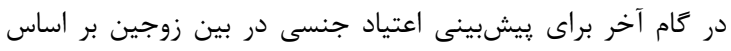

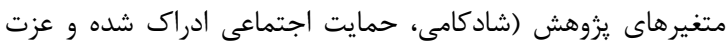

جدول ه: خلاصه نتايج تحليل ركرسيون جند مانه به روش همزمان جهت پيشبينى اعتياد جنسى زوجين

\begin{tabular}{|c|c|c|c|c|c|c|c|}
\hline $\mathbf{R}^{2}$ & $\mathbf{R}$ & Sig & $\mathbf{T}$ & $\beta$ & STE & B & متغير \\
\hline.$|9|$ & $\cdot 19 \mathrm{~V}$ &.$/ \cdot r$ & $-r / \cdot r$ & $-\cdot / \pi 4$ &.$/ 49$ & $-1 / 49$ & شادكامى \\
\hline$\cdot|9|$ & $\cdot 19 \mathrm{~V}$ & $\cdot / \cdot 99$ & $-r / 9 \Lambda$ & $-\cdot / r$ & $\cdot / \mu F$ &.$- / 94$ & حمايت خانواده \\
\hline.$|9|$ & $.19 \mathrm{~V}$ & $\cdot 1 \cdot \cdot \Delta$ & $-Y / A V$ & $-\cdot / T F$ & . AT & $-1 / T r$ & حمايت دوستان \\
\hline .191 & $\cdot 19 \mathrm{~V}$ & $\cdot|\cdot r|$ & $-T / T F$ &.$- / 19$ & $\cdot / \Delta T$ & $-1 / T F$ & حمايت ديكر افراد \\
\hline.$|9|$ & $\cdot 19 \mathrm{~V}$ & $\cdot 1 \cdot 4 \wedge$ & $-1 / 9 r$ & $-\cdot / 11$ & .1 .9 & $-\cdot / 1 \mathrm{~V}$ & عزت نفس \\
\hline
\end{tabular}

متغير ملاك: اعتياد جنسى

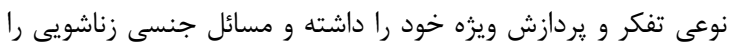

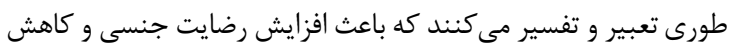
اعتياد جنسى آنان مى شود. همجنين نتايج نشان داد كه بين حمايت اجتماعى ادراك شده و اعتياد

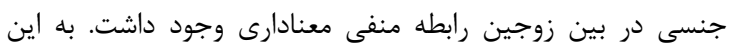

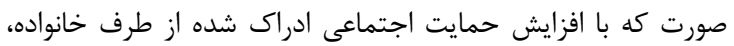

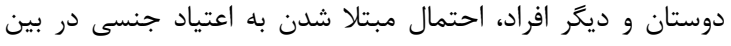

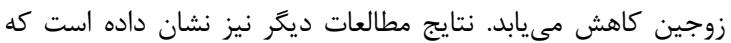

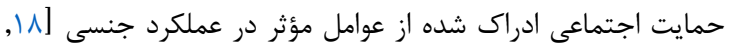

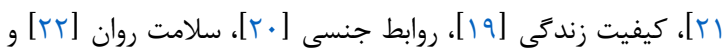

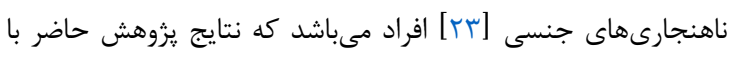

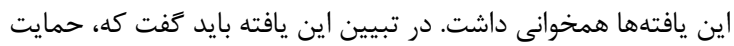

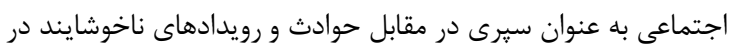

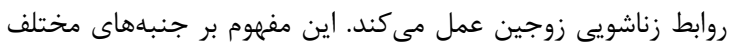

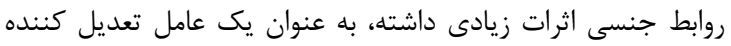

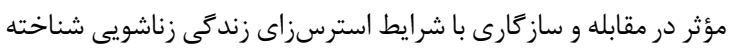

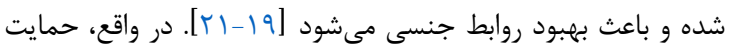

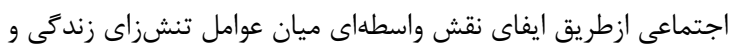

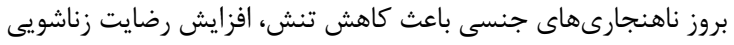

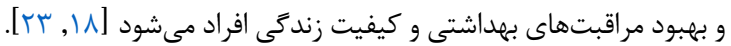

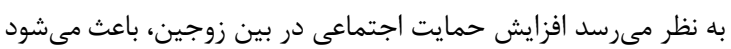

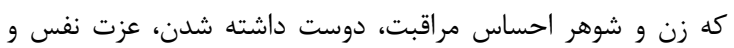

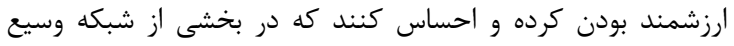

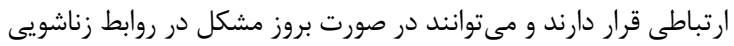

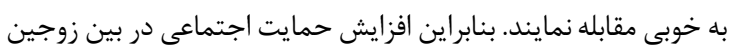

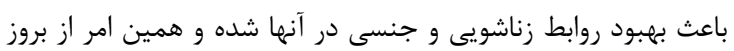
اعتياد جنسى جلوكيرى خواهد كرد.
نتايج كلى يزوهش حاضر نشان دهنده وجود روابط بين شادكامى،

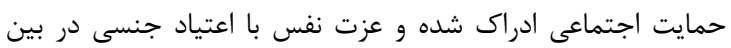

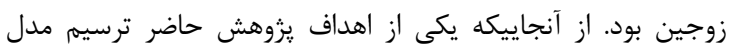

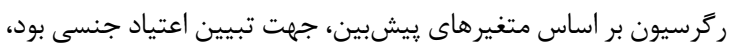

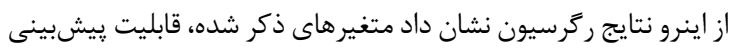

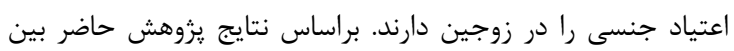

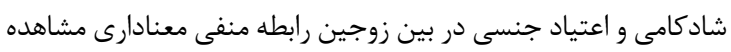

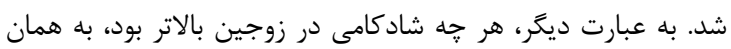

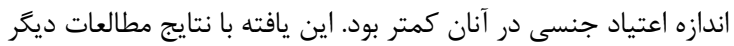

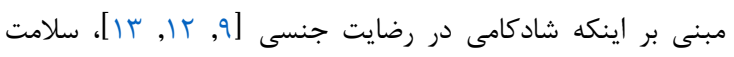

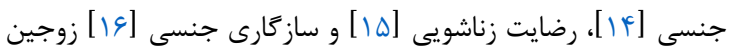

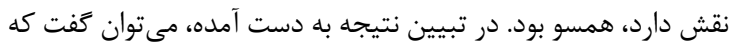

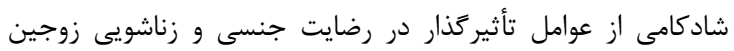

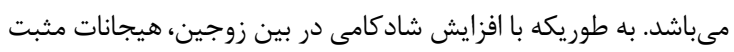

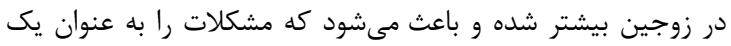

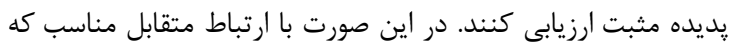

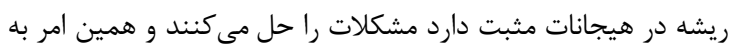

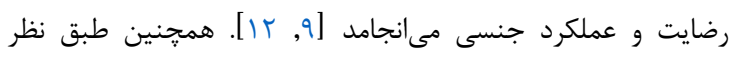
Bachmann , Rosen

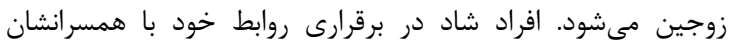

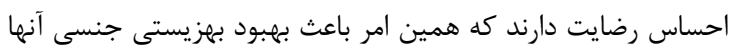

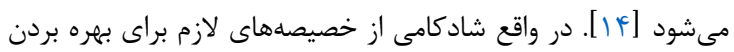

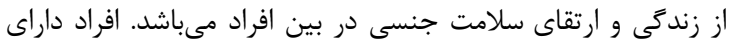

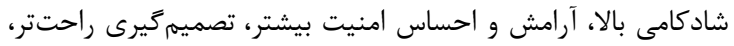

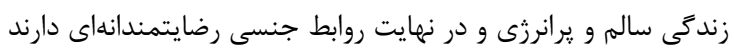

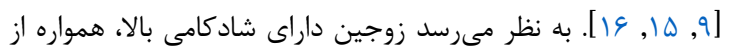

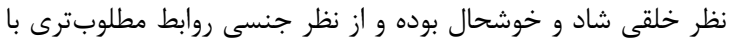

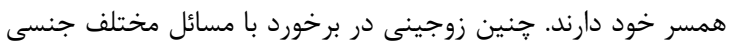




\section{نتيجه تيرى}

از اين مطالعه مىتوان نتيجه كرفت كه شادكامى، حمايت اجتماعى

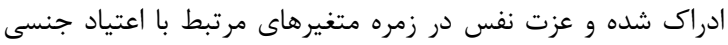

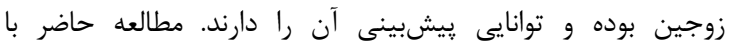

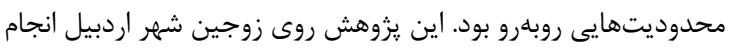

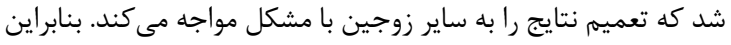

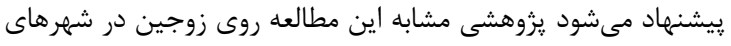

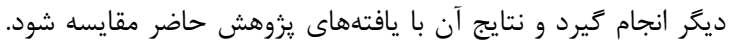

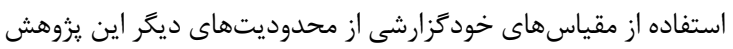

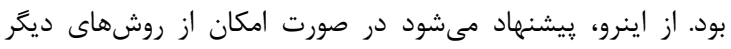

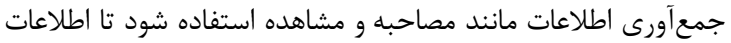

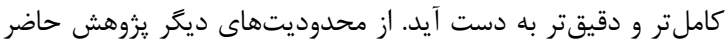

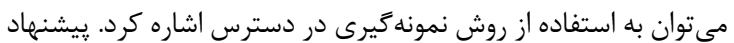

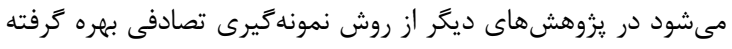

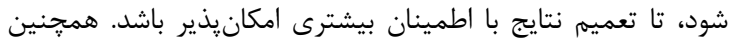

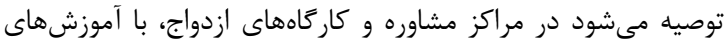
بهبود شادكامى، عزت نفس و همجنين افزايش حمايت اجتماعى، به دايه

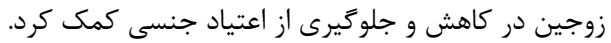

نويسندًان برخود لازم مى دانند از تمام زوجينى كه در اين مطالعه

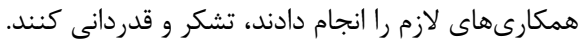

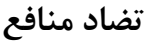

يزوهش حاضر هيج گَونه تضاد منافعى براى نويسندكان نداشته است.

\section{References}

1. Salehyan M, Aghabeiki A. The Effectiveness of CognitiveBehavioural Group Therapy on the Enhancement of the Self-Esteem of Women with Husbands Affected by Substance Dependency Disorder. Proc Soc Behav Sci 2011;30:1698-702. doi: 10.1016/j.sbspro.2011.10.328

2. Manwell LA, Barbic SP, Roberts K, Durisko Z, Lee C, Ware E, et al. What is mental health? Evidence towards a new definition from a mixed methods multidisciplinary international survey. BMJ Open. 2015;5(6):e007079. doi: 10.1136/bmjopen-2014-007079 pmid: 26038353

3. Samadifard H, Nariman M. [Prediction of Sexual Addiction of Couples Based on Meta-Cognitive Belief and Cognitive Fusion]. J Health Syst Res. 2018;14(2):183-8.

4. Sadock J, Kaplan L, Sadock A. Kaplan \& Sadock's Synopsis of Psychiatry-Behavioral Science/Clinical Psychiatry. Philadelphia: Worter Kluwer; 2007.

5. Zahediyan F, Mohammadi M, Samani S. [The Role of Attachment Styles, Parental Bonding and Self Concept in Sexual Addiction]. J Clin Psychol. 2011;3(3):65-73.

6. Kariman N, Sheikhan Z, Simbar M, Zahiroddin A, Akbarzadeh Bahgban A. [Sexual Dysfunction in Two Types of Hormonal Contraception: Combined Oral Contraceptives versus Depot Medroxy progesterone Acetate]. J Midwifery Reprod Health. 2017;5(1):806-13.

7. Hagedorn WB. Preparing Competent Clinicians: Curricular Applications Based on the Sexual Addiction Counseling Competencies. Sex Addict Compuls 2009;16(4):341-60. doi: 10.1080/10720160903399855

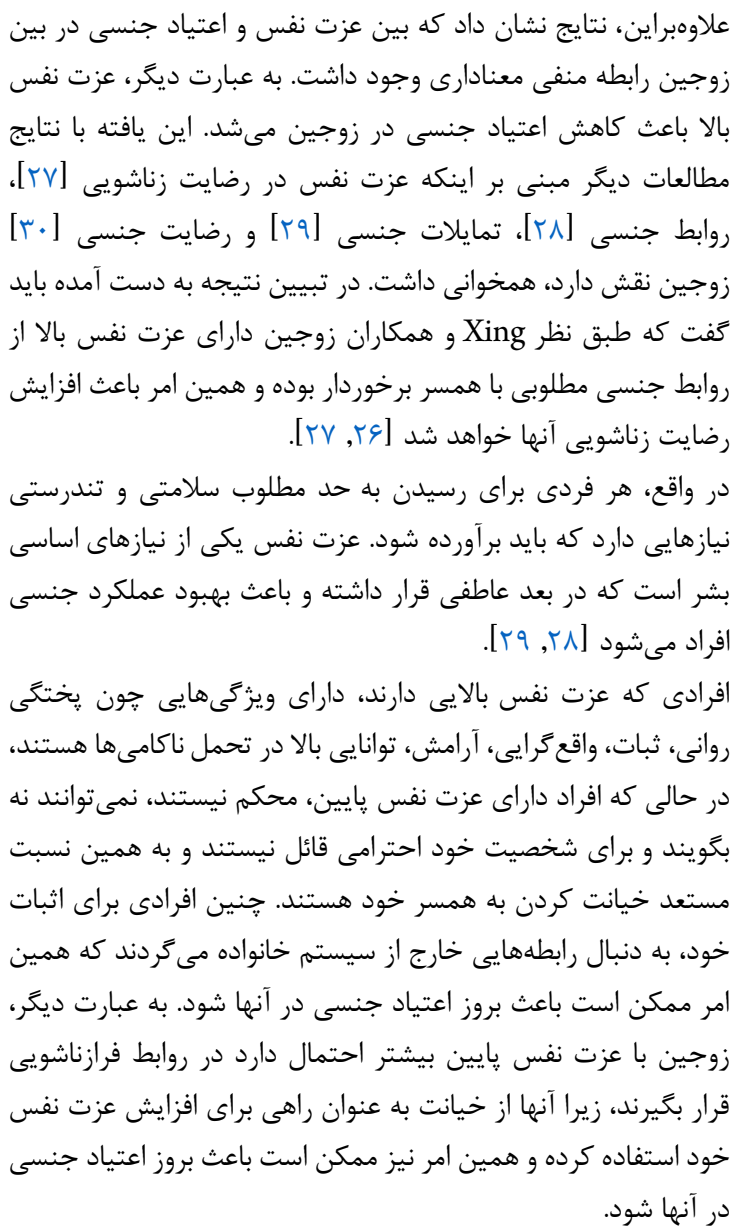

8. Briken P, Habermann N, Berner W, Hill A. Diagnosis and Treatment of Sexual Addiction: A Survey among German Sex Therapists. Sex Addict Compuls 2007;14(2):131-43. doi: 10.1080/10720160701310450

9. Heiman JR, Long JS, Smith SN, Fisher WA, Sand MS, Rosen RC. Sexual satisfaction and relationship happiness in midlife and older couples in five countries. Arch Sex Behav. 2011;40(4):741-53. doi: 10.1007/s10508-0109703-3 pmid: 21267644

10. Yazdani F, Kazemi A, Fooladi MM, Samani HR. The relations between marital quality, social support, social acceptance and coping strategies among the infertile Iranian couples. Eur J Obstet Gynecol Reprod Biol. 2016;200:58-62. doi: 10.1016/j.ejogrb.2016.02.034 pmid: 26972768

11. Kamiya Y, Akpalu B, Mahama E, Ayipah EK, OwusuAgyei S, Hodgson A, et al. The gender gap in relation to happiness and preferences in married couples after childbirth: evidence from a field experiment in rural Ghana. J Health Popul Nutr. 2017;36(1):8. doi: 10.1186/s41043-017-0084-2 pmid: 28298243

12. Fisher WA, Donahue KL, Long JS, Heiman JR, Rosen RC, Sand MS. Individual and Partner Correlates of Sexual Satisfaction and Relationship Happiness in Midlife Couples: Dyadic Analysis of the International Survey of Relationships. Arch Sex Behav. 2015;44(6):1609-20. doi: 10.1007/s10508-014-0426-8 pmid: 25370356 
13. Thomeer MB, Reczek C. Happiness and Sexual Minority Status. Arch Sex Behav. 2016;45(7):1745-58. doi: 10.1007/s10508-016-0737-z pmid: 27102605

14. Rosen RC, Bachmann GA. Sexual well-being, happiness, and satisfaction, in women: the case for a new conceptual paradigm. J Sex Marital Ther. 2008;34(4):291-7; discussion 8-307. doi: 10.1080/00926230802096234 pmid: 18576229

15. Sepehrian-Azar F, Mohammadi N, Badalpoor Z. [The Relationship between Hope and Happiness with Marital Satisfaction]. J Health Care. 2016;18(1):37-44.

16. Freak-Poli R, De Castro Lima G, Direk N, Jaspers L, Pitts M, Hofman A, et al. Happiness, rather than depression, is associated with sexual behaviour in partnered older adults. Age Ageing. 2017;46(1):101-7. doi: 10.1093/ageing/afw168 pmid: 28104602

17. Martins MV, Peterson BD, Almeida V, MesquitaGuimaraes J, Costa ME. Dyadic dynamics of perceived social support in couples facing infertility. Hum Reprod. 2014;29(1):83-9. doi: 10.1093/humrep/det403 pmid: 24218401

18. Littleton HL. The impact of social support and negative disclosure reactions on sexual assault victims: a crosssectional and longitudinal investigation. J Trauma Dissociation. 2010;11(2):210-27. doi: 10.1080/15299730903502946 pmid: 20373207

19. Shishehgar S, Mahmoodi A, Dolatian M, Mahmoodi Z, Bakhtiary M, Alavi Majd H. The Relationship of Social Support and Quality of Life with the Level of Stress in Pregnant Women Using the PATH Model. Iran Red Crescent Med J. 2013;15(7):560-5. doi: 10.5812/ircmj. 12174 pmid: 24396574

20. Golding JM, Wilsnack SC, Cooper ML. Sexual assault history and social support: six general population studies. J Trauma Stress. 2002;15(3):187-97. doi: 10.1023/A:1015247110020 pmid: 12092910

21. Fallah S, Dolatian M, Saberi S, Alavi-Majd H, Jabbari A, Mahmoodi Z. [The correlation of social support and sexual function of men with gender identity disorder after sex reassignment surgery]. J Health Promot Manage. 2016;5(4):8-15.

22. Ryan LH, Wan WH, Smith J. Spousal social support and strain: impacts on health in older couples. J Behav Med. 2014;37(6):1108-17. doi: 10.1007/s10865-014-9561-x pmid: 24622976

23. Buttram ME, Kurtz SP, Surratt HL. Substance use and sexual risk mediated by social support among Black men. J Community Health. 2013;38(1):62-9. doi: 10.1007/s10900-012-9582-8 pmid: 22752575

24. Salehi M, Kharaz Tavakol H, Shabani M, Ziaei T. The relationship between self-esteem and sexual self-concept in people with physical-motor disabilities. Iran Red Crescent Med J. 2015;17(1):e25359. doi: 10.5812/ircmj.25359 pmid: 25763279
25. Connors J, Casey P. Sex, body-esteem and self-esteem. Psychol Rep. 2006;98(3):699-704. doi: 10.2466/pr0.98.3.699-704 pmid: 16933665

26. Durmala J, Blicharska I, Drosdzol-Cop A, SkrzypulecPlinta V. The Level of Self-Esteem and Sexual Functioning in Women with Idiopathic Scoliosis: A Preliminary Study. Int J Environ Res Public Health. 2015;12(8):9444-53. doi: 10.3390/ijerph120809444 pmid: 26274967

27. Erol RY, Orth U. Development of self-esteem and relationship satisfaction in couples: Two longitudinal studies. Dev Psychol. 2014;50(9):2291-303. doi: 10.1037/a0037370 pmid: 24999764

28. Xing $X$, Pan $B C, D u$ Q Liang $X$, Wang XM, Wang $L$. [Impact of male infertility on men's self-esteem and satisfaction with sexual relationship]. Zhonghua Nan Ke Xue. 2013;19(3):223-7. pmid: 23700727

29. Wischmann T, Schilling K, Toth B, Rosner S, Strowitzki T, Wohlfarth K, et al. Sexuality, Self-Esteem and Partnership Quality in Infertile Women and Men. Geburtshilfe Frauenheilkd. 2014;74(8):759-63. doi: 10.1055/s-0034-1368461 pmid: 25221344

30. Ramezani M, Dolatian M, Shams J, Alavi H. [The relationship between self-esteem and sexual dysfunction and satisfaction in women]. Arak Med Univ J. 2012;14(59):57-65.

31. Carnes P. Don't call it love: Recovery from sexual addiction. Minneapolis, MN: Comp Care; 1991.

32. Cunningham J, McCrum Gardner E. Power, effect and sample size using G Power. Practical issues for researchers and members of research ethics committees. Evid Based Midwifery. 2007;5(4):132-6.

33. Argyle M, Lu L. The happiness of extraverts. Pers Individ Differ. 1990;11(10):1011-7. doi: 10.1016/01918869(90)90128-e

34. Alipour A, Agah Heris M. Reliability and validity of the oxford happiness inventory among Iranians. J Dev Psychol. 2007;3(12):287-98.

35. Zimet GD, Dahlem NW, Zimet SG, Farley GK. The Multidimensional Scale of Perceived Social Support. J Pers Assess 1988;52(1):30-41. doi: $10.1207 /$ s15327752jpa5201_2

36. Bruwer B, Emsley R, Kidd M, Lochner C, Seedat S. Psychometric properties of the Multidimensional Scale of Perceived Social Support in youth. Compr Psychiatry. 2008;49(2):195-201.

10.1016/j.comppsych.2007.09.002 pmid: 18243894

37. Salimi A, Jokar B, Nikpoor R. [Internet and communication: Perceived social support and Loneliness as antecedent variables]. Psychol Stud. 2009;5(3):81-102.

38. Coopersmith S. The antecedents of Self-esteem. San Fransisco: Freemand Company; 1967.

39. Pourdehghan M, Mohammadi S, Mahmmudnia A. [Relation between Self -Esteem and Marital Satisfaction among Guidance School Teachers]. J Appl Psychol. 2008;4(8):701-12. 